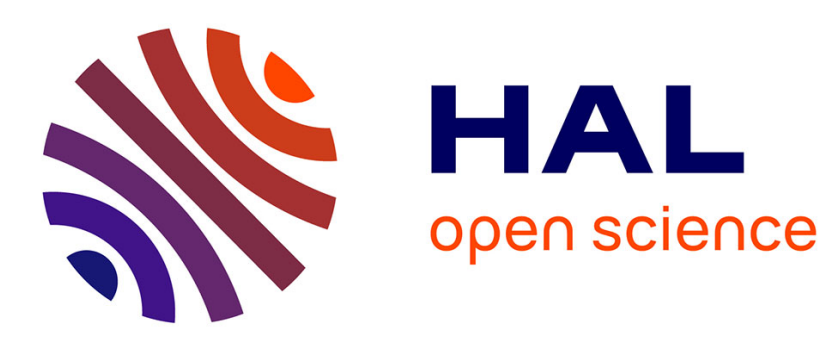

\title{
Integrating Environmental Temperature Conditions into the SIR Model for Vector-Borne Diseases
}

\author{
Md Arquam, Anurag Singh, Hocine Cherifi
}

\section{To cite this version:}

Md Arquam, Anurag Singh, Hocine Cherifi. Integrating Environmental Temperature Conditions into the SIR Model for Vector-Borne Diseases. Volume 2 Proceedings of the Eighth International Conference on Complex Networks and Their Applications COMPLEX NETWORKS 2019 Part of the Studies in Computational Intelligence book series (SCI, volume 882), pp.412-424, 2020, 10.1007/978-3-03036687-2_34.hal-02423853

\section{HAL Id: hal-02423853 https://hal.science/hal-02423853}

Submitted on 26 Dec 2019

HAL is a multi-disciplinary open access archive for the deposit and dissemination of scientific research documents, whether they are published or not. The documents may come from teaching and research institutions in France or abroad, or from public or private research centers.
L'archive ouverte pluridisciplinaire HAL, est destinée au dépôt et à la diffusion de documents scientifiques de niveau recherche, publiés ou non, émanant des établissements d'enseignement et de recherche français ou étrangers, des laboratoires publics ou privés. 


\title{
Integrating Environmental Temperature Conditions into the SIR Model for Vector-Borne Diseases
}

\author{
Md Arquam ${ }^{1}$, Anurag Singh ${ }^{2}$ and Hocine Cherifi ${ }^{3}$ \\ ${ }^{1,2}$ National Institute of Technology Delhi, New Delhi India \\ ${ }^{3}$ University of Burgundy, Dijon France \\ ${ }^{1}$ arquam@nitdelhi.ac.in, ${ }^{2}$ anuragsg@nitdelhi.ac.in, \\ ${ }^{3}$ hocine.cherifi@u-bourgogne.fr
}

\begin{abstract}
Nowadays, Complex networks are used to model and analyze various problems of real-life e.g. information diffusion in social networks, epidemic spreading in human population etc. Various epidemic spreading models are proposed for analyzing and understanding the spreading of infectious diseases in human contact networks. In classical epidemiological models, a susceptible person becomes infected after getting in contact with an infected person among the human population only. However, in vector-borne diseases, a human can be infected also by a living organism called a vector. The vector population that also help in spreading diseases is very sensitive to environmental factors such as temperature and humidity. Therefore, new researches are required to derive more realistic models to relate the dynamics of epidemics in the human population with environmental conditions. In order to integrate the impact of the temperature in the spreading of infection, we propose and investigate a modified SIR (Susceptible-Infected-Recovered) model tailored to vectorborne diseases. Simulations of the proposed model inspired by real datasets of infectious diseases are performed using an homogeneous human contact network. Results show that the proposed model corroborates the real-world data behavior, and it demonstrates its effectiveness to account for the temperature influence on the epidemic dynamics.
\end{abstract}

Keywords: Epidemic Spreading, Complex Network, Temperature, Dynamics on Network, SIR model

\section{Introduction}

More than $17 \%$ of all infectious diseases are vector-borne infections like dengue, cholera, small-pox which has been recognized in over 100 countries, and an estimated 50 - 100 million cases occur annually. The diseases are threatening about $60 \%$ of the world's population [1]. The disease is transmitted to the host population (human or animal) through physical contact with infectious hosts, or through infected vectors such as mosquitoes, ticks, flies, sandflies, fleas and bugs. The structure of the host population interactions, mobility and contacts 
patterns are key ingredients in the epidemic spreading process that find their best representation in the form of networks. Hence, network science is used to study the dynamics processes on network such as epidemic spreading in human networks [2]. The model has inspired many researchers in order to investigate the transmission dynamics of infectious diseases with various modifications. Esteva and Vargas proposed the SIR model to address dengue disease transmission considering constant and variable human populations [3]. Since then, many mathematical models have been proposed to study different aspects of infectious disease transmission. Pinho et al. used a mathematical model for dengue transmission analysis based on non-linear differential equations [4]. In this study, they performed a comparative analysis of epidemics that occurred in Salvador and Brazil, in 1995-1996 and 2002 showing the effect of vector control.

Vectors are generally sensitive to climatic factors. Indeed, weather influences survival and reproduction rates of vectors. One can note that the replication rates of mosquito-transmitted dengue viruses and malaria parasites increase at warmer temperatures [5]. Other factors such as habitat destruction, land use, pesticide application, and host density influence the vector activity (particularly biting rates) throughout the year. These assumptions provide a mathematical framework which connects the environmental conditions to vector-borne diseases. One of the principal components of the spreading of infectious disease is temperature, which is related to seasonal changes. Indeed, dengue, cholera and malaria are sensitive to climate change. Changing climate factors affect the potential for the spread of future vector-borne diseases. As temperature plays an important role in the behavior of the vectors, mathematical studies have been conducted to understand its influence in the transmission dynamics of vectorborne diseases. A large body of recent works concentrate on environmental data that affect vector populations to predict the epidemic process. These models do not explicitly include the host populations interactions and the underlying network structure, which also plays an important part in epidemic spreading $[6$, $2]$. Thus, rigorous studies are needed to understand the interplay between the various components of the epidemic processes : the host and vector population, the contact patterns and the environmental conditions. Such knowledge is essential to the development of novel and more effective intervention measures for vector-borne diseases.

In this paper, our main goal is to investigate the impact of environmental temperature on the spreading process of vector-borne diseases in the host population. Based on real-world data, we formulate the biting rate of vectors, and we derive the basic reproduction rate.

The rest of this paper is organized as follows: Section II discusses the related work on epidemic spreading. Section III describes the proposed model and section IV gives its mathematical formulation. Section V presents numerical simulations of the model and the analysis of the results. Finally, Section VI describes the conclusions and outlines future research directions. 


\section{Related Work}

In this section, we recall two surges of appropriate literature related to this work. First of all, we briefly recall important results about the influence of the environmental conditions on the vector population and its ability to transmit diseases to the host population. Then we turn to the epidemic spreading in the host population through its contact network. The most influential studies are related to SIR and SIS (Susceptible, Infected, Susceptible) models and the epidemic spreading in various types of the underlying contact network topology in the host population.

\subsection{Environmental factors of vector-borne epidemic spreading}

It is essential to get a good understanding of the potential of the vector to transmit diseases in various environmental conditions in order to predict accurately the underlying dynamic of vector-borne diseases in the host population. Therefore a large body of literature has been devoted to the complex biological and ecological relationships that exist between vectors and their environments. Liu-Helmersson et al. studied the vectorial capacity of Aedes aegypti and made investigations on the effects of temperature and implications for global dengue epidemic potential [7]. Polwiang discussed the seasonal basic reproduction of the number of dengue and impacts of climate on the transmission of the disease [8]. An epidemic model was proposed by Wang and Mulone to describe the dynamics of disease spread between two patches due to population dispersal. They proved that the reproduction number is a threshold of the uniform persistence and disappearance of the disease [9]. Dynamics of malaria disease was studied in the patchy environment by Auger et al. by using Ross-Macdonald model to $\mathrm{n}$-patches to describe the transmission dynamics of the disease [10].

\subsection{Contact network of the host population}

Due to the stochastic nature of epidemic spreading as it changes with time, different underlying network structures are used by various researchers to show the different spreading patterns. The advancement in the area of complex networks sets the base for the epidemic dynamics and initiated several related studies [11]. For example, a lot of emergent events in social networks and biological networks are pretended using the concept of complex networks [12]. Therefore, disease spreading patterns in the human population can be seen and analyzed by using the different topological structure [13].

Vespignani et al. [14] proposed the epidemic spreading model on the scale-free network to analyze the absence of epidemic threshold and its associated critical behaviour. Their proposal was based on computer virus spreading on communication and social networks. Moreno et al. [15] presented a new epidemiological framework characterized by a highly heterogeneous response of the system to the introduction of infected individuals with different connectivity considering the underlying scale-free network. Li et al. [16] proposed the general spreading dynamical behaviours in small-world evolving networks where control strategies are applied to suppress the propagation of diseases, viruses, and disasters. 
Significant gaps in the above studies and the necessity of further understanding of the effects of environmental variability on the dynamics of vector-host interactions inspire the present study. Temperature influences vector-borne diseases dynamics by affecting the dynamics of vector-host interactions. We present a dynamical system approach to explain the dynamics of vector-host interactions in order to integrate the impact of temperature. A model is developed by modifying the classical SIR model for the same purpose. The proposed work explains how adaptation occurs with temperature parameters, that can enable and respond to the occurrence of diseases, and how temperature-related parameters inhibit basic reproduction number.

\section{Description of the model}

Fig. 1 reports the number of infected persons for four different vector-born diseases, i.e. smallpox, malaria, dengue and typhoid. These data have been collected from Al-Shifa Hospital New Delhi during 2018 civil year. It appears that diseases spreading is slow in winter while in summer it is very high. This figure highlights the fact that temperature plays an important role in epidemic spreading. Corresponding average temperature and humidity are also plotted in Fig. 2. These figures suggest that the total number of infection increases after the rainy season. During this period, the weather is favourable for vector production. Indeed, rainfall increases the number and sizes of breeding sites, leading to an increase in the survival of juvenile stages of mosquitoes, a corresponding increase in the emergence rate of new adults, and a higher egg-laying rate and host-seeking behaviour in adults. Higher temperatures decrease the incubation period of the virus in mosquitoes, while very high or very low temperatures increase the mortality rate of mosquitoes. Fig. 2 shows that the temperature evolution throughout the year can be well approximated by a Gaussian shape ranging from $4^{\circ} \mathrm{C}$ to $34^{\circ} \mathrm{C}$. Note that previous models suggest that vector-borne diseases appear between $12^{\circ} \mathrm{C}$ and $34^{\circ} \mathrm{C}$ [7] [8].

Based on the analysis of the collected data from Al-Shifa Hospital and a literature review [8], we formulate the biting rate of a vector in term of temperature as follows:

$$
b(T)=b_{0} e^{-\left(T-T_{0}\right)^{2}}
$$

where $b_{0}$ is biting rate of vector at temperature $T_{0}$.

$T_{0}$ is the ambient temperature where biting is maximum. According to Polwiang [8] the maximum biting rate is equal to 0.4 at $25^{\circ} \mathrm{C}$.

We assume the following points for further formulation of the proposed model.

- The total host population of size $N_{h}$ follow the SIR model. It is subdivided into three classes: Susceptible $S_{h_{k}}(t)$, Infected $I_{h_{k}}(t)$, and Recovered $R_{h_{k}}(t)$

- The total vector population follow the SI model. It is subdivided into two classes: Susceptible $S_{v}$, and Infected $I_{v}$. Recovered class in the mosquito population is not considered due to their short lifespan. 


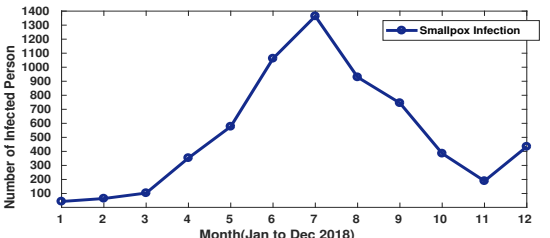

(a) Infection due to Smallpox each month

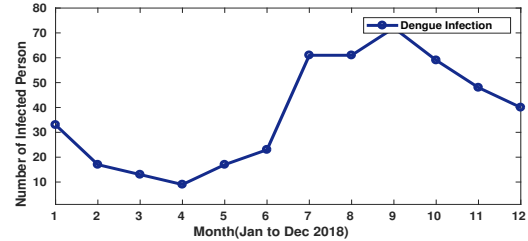

(c) Infection due to Dengue each month



(b) Infection due to Malaria in each month

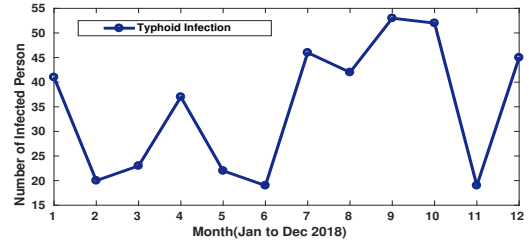

(c) Infection due to Typhoid each month

Fig. 1. Number of infected persons each month recorded at Al-shifa Hospital New Delhi for various diseases during the 2018 civil year

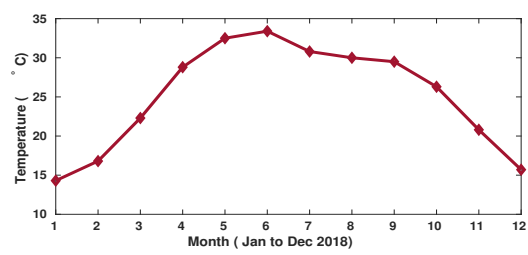

(a) Average Temperature of each month

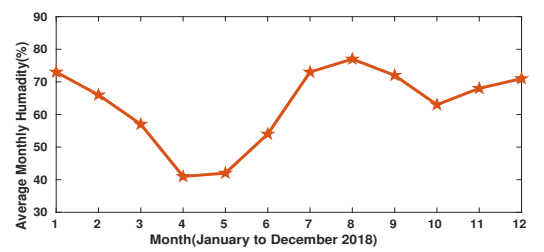

(b) Average humidity of each month

Fig. 2. Average temperature and humidity of Delhi-NCR monthwise during the 2018 civil year

- The Susceptible vectors are infected by biting infected hosts with rate of $\beta_{h v} b(T)$ where $\beta_{h v}$ is the spreading rate of disease from host to vector. $b(T)$ is the biting rate that depends on temperature.

- The Susceptible hosts are infected by biting of infected vectors with rate of $\beta_{v h} b(T)$ where $\beta_{v h}$ is the spreading rate of disease from vector to host.

- Infected hosts recover at the rate of $\mu_{h}$

- Susceptible host get infected by interaction with infected hosts at the rate of $\beta_{h}$, where $\beta_{h}$ is the spreading rate of disease from host to host.

Figure 3 represents a block diagram of the proposed model. It summarizes the interactions between vector and host as well as host to host interaction. A susceptible host may be infected by being bitten by an infected vector, and by interacting with an infected host. A susceptible vector can never be infected by interacting with an infected vector. A susceptible vector may become infected after biting an infected host. Both population are different, as population size of vector is much larger than host population. Both follow the different population dynamics as production rate as well as death rate of vectors are too high as 
compared to host population. Life span of vectors are also very small. Adaptive nature of vector towards environmental change is also not too strong. Therefore, change in environmental condition causes severe effect on vector population. Dynamics of both population are connected with each other by biting of vectors.



Layer 1:

Host Population

Fig. 3. Block diagram of the proposed model incorporating the temperature influence on the biting rate

\section{Mathematical formulation}

In this section, we integrate the effect of the environmental temperature conditions on the epidemic model. Let $\mathrm{G}(N, E)$ defines the network of $N$ nodes that represent the total host population and $E$ denotes the connections between nodes representing the interaction between individuals through which epidemic spreads. There are two type of population: host and vector population.

Let $N_{h}$ be the total host population, where $S_{h_{k}}(t), I_{h_{k}}(t)$ and $R_{h_{k}}(t)$ are the fraction of the Susceptible, Infected and Recovered nodes at time $t$ with degree $k$ with $S_{h_{k}}(t)+I_{h_{k}}(t)+R_{h_{k}}(t)=1$. Let $p(k)$ be the degree distribution of the network during the epidemic process, which describes the degree of the nodes where $k \in[1, N-1]$. The infection rate is given by $\beta_{h}$, and $\mu_{h}$ is the recovery rate in the host population. $\beta_{h}$ and $\mu_{h}$ are $\in[0,1]$. let $N_{v}$ be the vector population size and $S_{v}(t)$ and $I_{v}(t)$ are respectively the fraction of the susceptible and infected nodes at time $t$. The vector-borne diseases spread between hosts by direct contact and from host to vector or vector to host by vectors biting humans. For the sake of simplicity, we consider fixed populations sizes for both human and vector population. The transition rules of nodes from one state to another state is defined as follows:

- A healthy host node may become infected after getting in contact with an infected host node at time $t$ or by getting bitten by an infected vector.

- A healthy vector node may become infected by biting an infected host node.

- The vector population depends upon temperature hence biting also follow the same trend as biting depends on population.

- A host node may recover spontaneously at any time with rate $\mu_{h}$. Recovery of a node doesn't require any contact. Hence, we consider $\mu_{h}=1$, at each time stamp a host node recover.

- Once a node recover it never get infected again. 
- In addition, we are not considering demography for the host and vector population. Therefore, the total number of hosts and vectors are constant throughout the transition.

Based on the above transitions for homogeneous networks, the mean-field rate equations are defined as follows:

$$
\begin{aligned}
\frac{d S_{h_{k}}(t)}{d t} & =-\beta_{h}\langle k\rangle S_{h_{k}}(t) I_{h_{k}}(t)-\beta_{v h} b(T) S_{h_{k}}(t) I_{v}(t) \\
\frac{d I_{h_{k}}(t)}{d t}=\beta_{h}\langle k\rangle S_{h_{k}}(t) I_{h_{k}}(t) & +\beta_{v h} b(T) S_{h_{k}}(t) I_{v}(t)-\mu_{h} I_{h_{k}}(t) \\
\frac{d R_{h_{k}}(t)}{d t} & =\mu_{h} I_{h_{k}}(t)
\end{aligned}
$$

We always consider $\frac{d N_{h}}{d t}=0$ and $\frac{d N_{v}}{d t}=0$

$$
\begin{aligned}
& \frac{d S_{v}(t)}{d t}=-\beta_{h v} b(T) S_{v}(t) I_{h_{k}}(t)-\mu_{v} S_{v}(t) \\
& \frac{d I_{v}(t)}{d t}=\beta_{h v} b(T) S_{v}(t) I_{h_{k}}(t)-\mu_{v} I_{v}(t)
\end{aligned}
$$

Table 1. Description of the parameters used in the model

\begin{tabular}{|c|c|}
\hline Name of Parameter & Interpretation \\
\hline$S_{h_{k}}$ & Fraction of Susceptible host \\
\hline$I_{h_{k}}$ & Fraction of Infected host \\
\hline$R_{h_{k}}$ & Fraction of Recovered host \\
\hline$S_{v}$ & Fraction of Susceptible Vector \\
\hline$I_{v}$ & Fraction of Infected Vector \\
\hline$\beta_{h}$ & Transmission probability from host to host \\
\hline$\beta_{v h}$ & Transmission probability from vector to host \\
\hline$\beta_{h v}$ & Transmission probability from host to vector \\
\hline$b(T)$ & Temperature dependent biting rate of vector \\
\hline$\mu_{v}$ & Death rate of vector population \\
\hline$\mu_{h}$ & Recovery rate of host population \\
\hline
\end{tabular}

An epidemic occurs if the number of infected vectors increases, i.e., $\frac{d I_{v}(t)}{d t}>0$, Hence, from equation 6 ,

$$
\begin{array}{r}
\frac{d I_{v}(t)}{d t}>0 \\
\beta_{h v} b(T) S_{v}(t) I_{h_{k}}(t)-\mu_{v} I_{v}(t)>0 \\
I_{v}(t)<\frac{\beta_{h v} b(T) S_{v}(t) I_{h_{k}}(t)}{\mu_{v}}
\end{array}
$$

At the outset of an epidemic, $S_{v}(t) \approx 1$. Death is an instantaneous process, therefore $\mu_{v}=1$ then, $\beta_{h v} b(T) I_{h_{k}}(t)>I_{v}(t)$. This should be greater than one. The basic reproduction number of vector is given by: 
$R_{0_{v}}=\beta_{h v} b(T) I_{h_{k}}(t)$.

By reporting the value of $I_{v}(t)$ into equation 2 and 3 , the above equation can be written:

$$
\begin{gathered}
\frac{d S_{h_{k}}(t)}{d t}=-\beta_{h}\langle k\rangle S_{h_{k}}(t) I_{h_{k}}(t)-\beta_{v h} b(T) S_{h_{k}}(t)\left\{\beta_{h v} b(T) I_{h_{k}}(t)\right\} \\
\frac{d I_{h_{k}}(t)}{d t}=\beta_{h}\langle k\rangle S_{h_{k}}(t) I_{h_{k}}(t)+\beta_{v h} b(T) S_{h_{k}}(t)\left\{\beta_{h v} b(T) I_{h_{k}}(t)\right\}-I_{h_{k}}(t) \\
\frac{d R_{h_{k}}(t)}{d t}
\end{gathered}
$$

Generally, a healthy host node is infected, and this infected node is converted into a recovered node. So we can say $S_{h_{k}}(t)$ is converted into $R_{h_{k}}(t)$. Therefore, from Eq.8 and Eq.10,

$$
\frac{d S_{h_{k}}(t)}{d R_{h_{k}}(t)}=\frac{-\left(\beta_{h}\langle k\rangle S_{k_{h}}(t)+\beta_{v h} b(T) S_{h_{k}}(t) \beta_{h v} b(T) I_{h_{k}}(t)\right.}{I_{h_{k}}(t)}
$$

where, Eq. 11 shows the rate of change of susceptible nodes to recovered nodes. Integrating both side of Eq.11

$$
S_{k_{h}}(t)=e^{-\left(\beta_{h}\langle k\rangle+\beta_{v h} b(T)^{2} \beta_{h v}\right) R_{h_{k}}(t)}
$$

The negative exponent in Eq. 12 shows that the number of susceptible nodes is decreasing and converted into recovered nodes. Epidemic reaches a steady state at $t \rightarrow \infty$ hence, $I_{h_{k}}(\infty)=0$. Therefore, the normalized condition for the steady state is

$$
\begin{aligned}
& S_{k_{h}}(\infty)=e^{-\left(\beta_{h}\langle k\rangle+\beta_{v h} b(T)^{2} \beta_{h v}\right) R_{h_{k}}(\infty)} \\
& R_{h_{k}}(\infty)=1-e^{-\left(\beta_{h}\langle k\rangle+\beta_{v h} b(T)^{2} \beta_{h v}\right) R_{h_{k}}(\infty)}
\end{aligned}
$$

Now let $f\left(R_{h_{k}}(\infty)\right)=1-e^{-\left(\beta_{h}\langle k\rangle+\beta_{v h} b(T)^{2} \beta_{h v}\right) R_{h_{k}}(\infty)}$ be a function of $R_{h_{k}}(\infty)$ and strictly increasing. If we put $R_{h_{k}}(\infty)=0$, then the whole population of host recover and it gives us a trivial solution. It also explains about disease free state.

Now we need to find some non trivial solution which lies between 0 and 1 . For this the following condition must satisfy

$$
\begin{gathered}
\left.\frac{d f\left(R_{h_{k}}(\infty)\right)}{d R_{h_{k}}(\infty)}\right|_{R_{h_{k}}(\infty)=0}>1 \\
\left.\left(\beta_{h}\langle k\rangle+\beta_{v h} b(T)^{2} \beta_{h v}\right) e^{-\left(\beta_{h}\langle k\rangle+\beta_{v h} b(T)^{2} \beta_{h v}\right) R_{h_{k}}(\infty)}\right|_{R_{h_{k}}(\infty)=0}>1 \\
\left(\beta_{h}\langle k\rangle+\beta_{v h} b(T)^{2} \beta_{h v}\right)>1
\end{gathered}
$$

Now, we can say that basic reproduction $R_{0_{h}}$ must be $\left(\beta_{h}\langle k\rangle+\beta_{v h} b(T)^{2} \beta_{h v}\right)>1$ to spread the epidemic in the host population.

Therefore,

$$
R_{0_{h}}=\left(\beta_{h}\langle k\rangle+\beta_{v h} b(T)^{2} \beta_{h v}\right)
$$

where $\beta_{h},\langle k\rangle, \beta_{v h}$ and $\beta_{h v}$ are constant. Hence, $R_{0_{h}}$ is directly proportional to the square of biting rate i.e. $b(T)^{2}$. This basic reproduction rate is also called the critical threshold of spreading of disease. 


\section{Simulation of the model and results analysis}

In this section, we first report the simulation setup, and then we discuss the results of the simulation performed using the temperature dependent SIR model using a homogeneous network (WattsStrogatz model) as the underlying contact network. The various parameters values used for simulations are listed in Table 2. These values have been chosen according to a literature review

Table 2. Parameters values used in the simulation

\begin{tabular}{|c|c|}
\hline Name of Parameter & Value \\
\hline Host contact network size & 2000 \\
\hline Connectivity probability for WattsStrogatz model & 0.2 \\
\hline Number of neighbour of each node & 300 \\
\hline Vector population size & 100000 \\
\hline Spreading Rate between host to host $\left(\beta_{h}\right)$ & 0.6 \\
\hline Recovery Rate $\left(\mu_{h}\right)$ & 1 \\
\hline Death Rate of vector $\left(\mu_{v}\right)$ & 1 \\
\hline Spreading Rate between vector to host $\left(\beta_{v h}\right)$ & 0.4 \\
\hline Biting rate of vector $\left(b_{0}\right)$ at $T_{0}$ & 0.6 \\
\hline$T_{0}$ & 0.4 \\
\hline Range of temperature $(T)$ & $25^{\circ} \mathrm{C}$ \\
\hline
\end{tabular}

We focus on the effect of temperature on the dynamics of epidemics on the host contact network as well as the vector population. In the simulation, if the temperature is in the range $T<0^{\circ} \mathrm{C}$ or $T>37^{\circ} \mathrm{C}$, the vector biting rate is zero. In other words, outside the limit temperatures, no vectors are present. Within that range of temperature the critical threshold is given by:

$$
R_{0_{h}}=\left(\beta_{h}\langle k\rangle+\beta_{v h} b(T)^{2} \beta_{h v}\right)
$$

The epidemic spreading with the modified SIR Model on homogeneous network as underlying topology (WattsStrogatz model) is shown in Fig. 4. We took the value of temperature $T$ ranging from $4.3^{\circ} \mathrm{C}$ to $37^{\circ} \mathrm{C}$ to analyze the effect of temperature in the infection process (temperature of Delhi NCR in 2018). The epidemic spreading evolution of the SIR spreading model for the host population is reported in Fig. 4(a). Similar results for the vector population is shown in Fig. 4(b). These figures show that the infection increases with time until the optimum temperature is reached. After that, the infection starts decreasing. The timespan of the existence of epidemic depends upon the existence of the vector population as shown in Fig. 4(b).

One can observe that the vector population vanished as much as quick due to short life span but it increases the epidemic threshold as mentioned in Fig.5. Fig.5(a)\&(b) illustrate the evolution in the epidemic threshold in the vector population with the temperature variation. Fig.5(c)\&(d) present also the variation of the epidemic threshold but in the host population with a change in temperature. The infection threshold varies from 0.8 to more than 0.9 in the host population while it does not change a lot in the vector population because the life span 


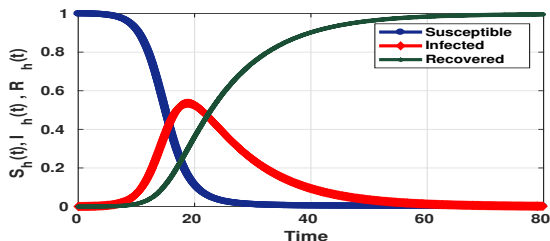

(a) Spreading in Host population

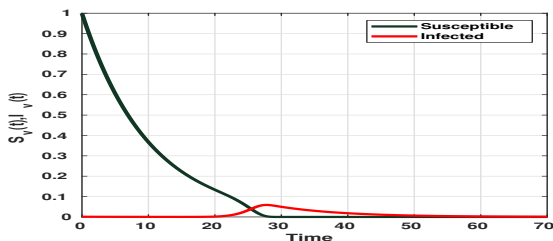

(b) Spreading in vector population

Fig. 4. Epidemic Spreading in Host and Vector Population

of the vector is very small. These results corroborate researches reported in the literature that have already proved that the transmission probability from host to vector is greater than transmission probability from vector to host.
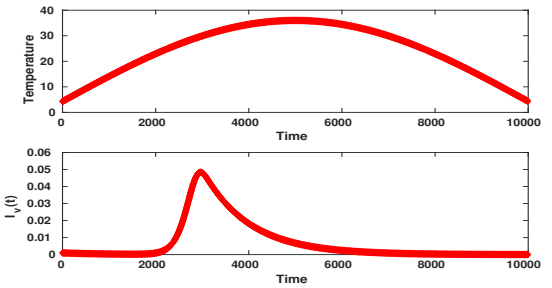

(a) Infection threshold of vector population
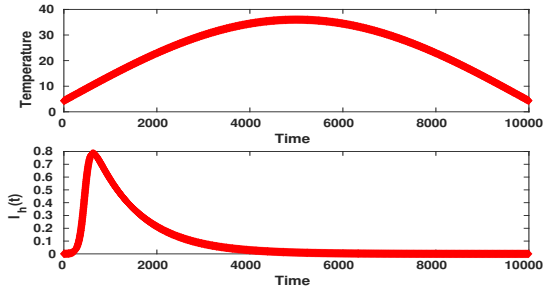

(c) Infection threshold in host population


(b) Infection threshold of vector population
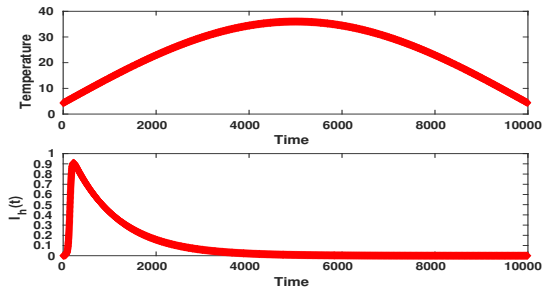

(d) Infection threshold in host population

Fig. 5. Effect of temperature on infection threshold in SIR considering a homogeneous contact network

We also analyse the effect of temperature on the biting rate that depends upon the total population of vectors. As temperature increases from $25^{\circ} \mathrm{C}$, then mosquitoes start biting till the maximum temperature. After that once temperature reaches $37^{\circ} \mathrm{C}$ then biting becomes null as the vector population vanish. The biting rate is plotted in Fig. 6. Fig. 6(a) shows that biting is maximum at the middle of the spreading process, while Fig. 6(b) shows that biting increases with the increase of temperature till ambient temperature. After that, the vector population starts dying.Finally, after reaching the maximum temperature the vector population is eliminated.

Fig. 7 explains the effect of temperature on infection spreading in vector as well as the host population. An infected vector can cause infection in multiple 


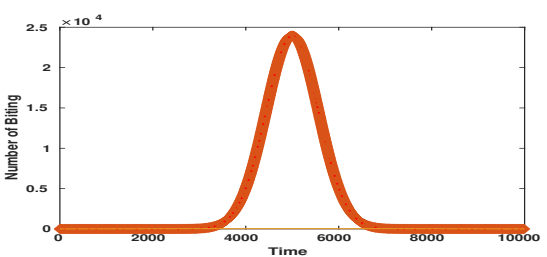

(a) Total biting of vectors

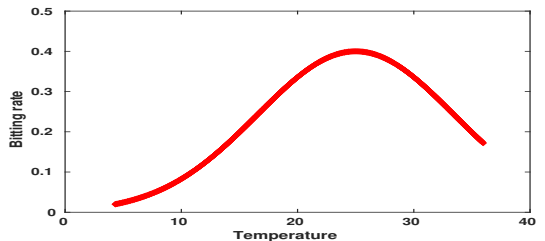

(b) Biting rate of vector population versus temperature

Fig. 6. Biting of vector population

hosts. Vector population is much larger than the host population. Therefore, infection in the host population increases more than in vector population.

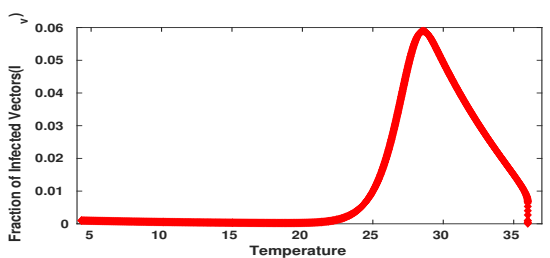

(a) Infection spreading in vector versus temperature



(b) Infection spreading in host versus temperature

Fig. 7. Effect of Temperature on infection spreading in vector and host populations

\section{Conclusion and Future work}

In this work, we propose and investigate a modified SIR model which integrates the effect of temperature on spreading of vector-borne diseases. Here, we consider two type of populations: 1) the host population with the three states of the SIR Model and 2) the vector population with the two states of the SI Model. Favourable temperature increases the disease spreading from vector to host and by cascading effect to the host population. We show that the threshold of spreading rate of the disease is proportional to the square of the biting rate $(b(T))$ which is defined as the function of temperature. Simulations are performed using the proposed modified SIR model using an homogeneous contact network. They show that temperature increases the critical threshold value of the spreading rate. Additionally, if the temperature increases above $37^{\circ} \mathrm{C}$, the epidemic die out due to the extinction of the vector population. Result of real data of diseases are plotted, which shows similar infection pattern in host population. We plan to develop this work in various future directions. An important extension is to include the effect of humidity in our future studies as most diseases spread after the rainy season in India especially. Furthermore more realistic scenario need to be considered concerning the host contact network topology such as scale-free networks, modular networks and dynamic networks [17] [18] [19]. The movement of population may also be considered.

\section{References}

1. World Health Organization et al. Global strategy for dengue prevention and control 2012-2020. 2012. 
2. Roy M Anderson, Robert M May, and B Anderson. Infectious diseases of humans: dynamics and control, volume 28. Wiley Online Library, 1992.

3. Lourdes Esteva and Cristobal Vargas. Analysis of a dengue disease transmission model. Mathematical biosciences, 150(2):131-151, 1998.

4. Suani Tavares Rubim de Pinho, Claudia P Ferreira, Lourdes Esteva, Florisneide Rodrigues Barreto, VC Morato e Silva, and MGL Teixeira. Modelling the dynamics of dengue real epidemics. Philosophical Transactions of the Royal Society A: Mathematical, Physical and Engineering Sciences, 368(1933):5679-5693, 2010.

5. Dana A Focks, Eric Daniels, Dan G Haile, and James E Keesling. A simulation model of the epidemiology of urban dengue fever: literature analysis, model development, preliminary validation, and samples of simulation results. The American journal of tropical medicine and hygiene, 53(5):489-506, 1995.

6. Premeshori Waikhom, Renu Jain, and Sandeep Tegar. Sensitivity and stability analysis of a delayed stochastic epidemic model with temperature gradients. Modeling Earth Systems and Environment, 2(1):49, 2016.

7. Jing Liu-Helmersson, Hans Stenlund, Annelies Wilder-Smith, and Joacim Rocklöv. Vectorial capacity of aedes aegypti: effects of temperature and implications for global dengue epidemic potential. PloS one, 9(3):e89783, 2014.

8. Sittisede Polwiang. The seasonal reproduction number of dengue fever: impacts of climate on transmission. PeerJ, 3:e1069, 2015.

9. Wendi Wang and G Mulone. Threshold of disease transmission in a patch environment. Journal of Mathematical Analysis and Applications, 285(1):321-335, 2003.

10. Pierre Auger, Etienne Kouokam, Gauthier Sallet, Maurice Tchuente, and Berge Tsanou. The ross-macdonald model in a patchy environment. Mathematical biosciences, 216(2):123-131, 2008.

11. Maziar Nekovee, Yamir Moreno, Ginestra Bianconi, and Matteo Marsili. Theory of rumour spreading in complex social networks. Physica A: Statistical Mechanics and its Applications, 374(1):457-470, 2007.

12. Réka Albert and Albert-László Barabási. Statistical mechanics of complex networks. Reviews of modern physics, 74(1):47, 2002.

13. Romualdo Pastor-Satorras, Claudio Castellano, Piet Van Mieghem, and Alessandro Vespignani. Epidemic processes in complex networks. Reviews of modern physics, 87(3):925, 2015.

14. Alessandro Vespignani. Modelling dynamical processes in complex socio-technical systems. Nature physics, 8(1):32, 2012.

15. Yamir Moreno, Romualdo Pastor-Satorras, and Alessandro Vespignani. Epidemic outbreaks in complex heterogeneous networks. The European Physical Journal B-Condensed Matter and Complex Systems, 26(4):521-529, 2002.

16. Xiang Li and Xiaofan Wang. Controlling the spreading in small-world evolving networks: stability, oscillation, and topology. IEEE Transactions on Automatic Control, 51(3):534-540, 2006.

17. Keziban Orman, Vincent Labatut, and Hocine Cherifi. An empirical study of the relation between community structure and transitivity. Menezes R., Evsukoff A., Gonzlez M. (eds) Complex Networks. Studies in Computational Intelligence, 424:99-110, 2013.

18. Navin Gupta, Anurag Singh, and Hocine Cherifi. Centrality measures for networks with community structure. Physica A: Statistical Mechanics and its Applications, 452:46-59, 2016.

19. Zakaryia Ghalmane, Mohammed El Hassouni, Chantal Cherifi, and Hocine Cherifi. Centrality in modular networks. EPJ Data Science, 8(15), 2019. 